\title{
Conformational analysis and vibrational spectroscopic investigation of L-alanyl-L-glutamine dipeptide
}

\author{
Serda Kecel ${ }^{\mathrm{a}, *}$, Aysen E. Ozel ${ }^{\mathrm{a}}$, Sevim Akyuz ${ }^{\mathrm{b}}$ and Sefa Celik ${ }^{\mathrm{c}}$ \\ ${ }^{a}$ Faculty of Sciences, Department of Physics, Istanbul University, Istanbul, Turkey \\ ${ }^{\mathrm{b}}$ Faculty of Science and Letters, Department of Physics, Istanbul Kultur University, Atakoy Campus, \\ Istanbul, Turkey \\ ${ }^{\mathrm{c}}$ Engineering Faculty, Department of Electrical-Electronics Engineering, Istanbul University, Avcilar, \\ Istanbul, Turkey
}

\begin{abstract}
In this study conformational behavior of anticancer chemotherapy dipeptide Ala-Gln and its dimers have been investigated by molecular mechanic and ab-initio calculations. The calculations on Ala-Gln dipeptide as a function of side chain torsion angles, enable us to determine their energetically preferred conformations. The relative positions of the side chain residues of the stable conformations of dipeptide were obtained, depending on the obtained conformational analysis results. The lowest energy conformation of the dipeptide has been determined by using the Ramachandran maps (Biopolymers 6 (1963), 1494; J. Mol. Biol. 7 (1963), 95) and compared with the quantum chemical ab-initio results. The geometry optimization, vibrational wavenumbers and intensity calculations of Ala-Gln dipeptide were carried out with the Gaussian03 program by using DFT with B3LYP functional and 6-31++G(d,p) basis set. The IR $\left(4000-400 \mathrm{~cm}^{-1}\right)$ and Raman spectra of the Ala-Gln dipeptide have been reported in solid phase, and compared with the theoretical vibrational data.
\end{abstract}

Keywords: Conformational analysis, IR and Raman spectra, DFT calculations, L-alanyl-L-glutamine dipeptide

\section{Introduction}

It is known that the biological functions of the peptides in living systems are related with their threedimensional structures. Conformational properties of the biologically active peptides are very important for investigation of their functional structure. Besides, electronic and vibrational properties of a molecule are related with its conformational structure.

Glutamine plays an important role in the immune system. It is essential for cell proliferation, that it can act as a respiratory fuel and that it can enhance the function of stimulated immune cells [3]. Oral supplementation with glutamine can significantly decrease the severity of chemotherapy-induced stomatitis, an important cause of morbidity in the treatment of patients with cancer [14]. On the other hand, glutamine supplementation may reduce the incidence of gastrointestinal, neurologic and possibly cardiac complications of cancer therapy [2,13]. Moreover, glutamine depletion has been observed in acute pancreatitis and may contribute to the morbidity of this disease [9].

\footnotetext{
${ }^{*}$ Corresponding author: S. Kecel, Faculty of Sciences, Department of Physics, Istanbul University, Vezneciler 34134, Istanbul, Turkey. E-mail: skecel@istanbul.edu.tr.
} 
L-alanyl-L-glutamine (Ala-Gln) dipeptide is know to reduce chemotherapy-induced side-effects, and has demonstrated immunomodulatory, anticatabolic/anabolic, gastrointestinal mucosal protective and antioxidant activities [15]. It is used to prevent of mucositis in patients with head-and-neck cancer [1]. In spite of the above-mentioned importance, up to our knowledge, no study on conformational analysis and quantum chemical calculations on Ala-Gln dipeptide reported yet. In this work, the results of conformational analysis and the experimental and theoretical IR and Raman data of Ala-Gln dipeptide are reported. The theoretical conformational analysis methods allow us to determine whole sets of energetically preferred conformers of peptide molecule. The vibrational wavenumbers of dimers of L-alanyl-L-glutamine dipeptide have also been calculated.

\section{Experimental and computational details}

The solid Ala-Gln dipeptide was purchased from Sigma Aldrich Co. Ltd. (Cas: 39537-23), and used as received. The IR spectra of $\mathrm{KBr}$ discs were recorded on a Jasco 300E FT-IR spectrometer $\left(2 \mathrm{~cm}^{-1}\right.$ resolution) between $400-4000 \mathrm{~cm}^{-1}$ spectral region. The Raman spectra of the samples were taken with a Jasco NRS-3100 micro-Raman spectrometer (1800 lines/mm grating and high sensitivity cooled CCD). The spectrometer was calibrated with the silicon phonon mode at $520 \mathrm{~cm}^{-1}$. Either 532 or $785 \mathrm{~nm}$ lines of the diode lasers was used for excitation. The exposure time was taken as $2 \mathrm{~s}$ and 100 spectra were accumulated. Spectral resolution was better than $4 \mathrm{~cm}^{-1}$.

The conformational analysis of dipeptide was carried out by sequential method with combining all low-energy conformations of constitutive residues. The conformational potential energy of a molecule is given as the sum of the independent contributions of nonbonded $\left(E_{\mathrm{nb}}\right)$, electrostatic $\left(E_{\mathrm{el}}\right)$, torsional interactions $\left(E_{\text {tors }}\right)$ and hydrogen bonds $\left(E_{\mathrm{hb}}\right)$ energies and then the conformational energy was minimized using program proposed by Godjaev et al. in FORTRAN [6]. The obtained results for the global conformation were used as initial values for geometry optimization performed by DFT/B3LYP.

Due to the success in calculating the electronic structure and energy, the calculations were carried out by using the hybrid density functional theory (DFT/B3LYP) method, either with the complete basic sets $\{6-31 \mathrm{G}(\mathrm{d}), 6-31 \mathrm{G}(\mathrm{d}, \mathrm{p})$ and 6-31G $++(\mathrm{d}, \mathrm{p})\}$ (for monomer) or with only 6-31G(d,p) (for dimer). All computational studies were carried out with the Gaussian03 package program [4].

The Total Energy Distribution (TED) of the vibrational modes of the molecules was calculated by using Parallel Quantum Mechanics Solutions (PQS) program [10] and the fundamental vibrational modes were characterised by their total energy distribution. The differences between the calculated and the corresponding experimental values are often attributed to the neglect of anharmonicity and incomplete inclusion of electronic correlation effects. In order to correct overestimation between unscaled wavenumbers and observed wavenumbers, dual scaling factors were used. The wavenumbers under $1800 \mathrm{~cm}^{-1}$, were scaled either with 0.967 (for B3LYP/6-31G(d,p)) or 0.977 (for B3LYP/6-31++G(d,p)), and for over $1800 \mathrm{~cm}^{-1}$ the scale factor 0.955 were used for both B3LYP/6-31G(d,p), B3LYP/6-31++G(d,p) levels of theory.

\section{Result and discussion}

The objective of the first part of this study is to report the result of the theoretical conformational studies on alanine-glutamine dipeptide, in order to determine the stable conformers. In the second part of this study, the geometry optimization of the global conformation was performed and after then the 
vibrational wavenumbers were calculated. The same calculations were repeated for the dimeric forms of the dipeptide.

The molecular model of the neutral form of Ala-Gln dipeptide with the atom numbering was given in Fig. 1(a). The starting conformations of the dipeptide Ala-Gln were obtained by combining the low energy structures of constitutive residues. The theoretical conformational analysis methods allow us to determine the whole sets of energetically preferred conformers of the dipeptide molecule. The obtained values of the dihedral angles of the side chains of the global conformation of neutral Ala-Gln dipeptide are tabulated in Table 1(a). The conformational potential energy of global conformation of Ala-Gln molecule $\left(E_{\mathrm{tot}}=-0.18 \mathrm{kcal} / \mathrm{mol}\right)$ is given as the sum of the van der Waals $\left(E_{\mathrm{vdW}}=-6.06 \mathrm{kcal} / \mathrm{mol}\right)$, electrostatic $\left(E_{\mathrm{el}}=4.41 \mathrm{kcal} / \mathrm{mol}\right)$, and torsional interactions $\left(E_{\mathrm{tor}}=1.47 \mathrm{kcal} / \mathrm{mol}\right)$ energies. Knowledge of the conformations of peptides mainly concerns the study of their biological functions and has very significant role on their interactions and their chemical activities. To provide a guide for the assignment of the vibrational spectra, geometry optimization studies were performed for non-zwitterionic form of the lowest energy conformer of Ala-Gln. Calculated bond distances, interbond angles and torsion angles of mono Ala-Gln and dimer I are tabulated in Table 1(b). On the basis of the calculation results, two possible intra $\mathrm{H}$-bonding interactions between $\mathrm{H}(3)$ and $\mathrm{O}(23)(1.95 \AA), \mathrm{H}(13)$ and $\mathrm{O}(23)$ $(2.25 \AA)$ were predicted for the global conformation of monomeric Ala-Gln dipeptide. The dimeric forms of Ala-Gln dipeptite were constructed by bringing together two identical Ala-Gln monomers in possible configurations, as a result of geometry optimization studies, four low energy dimeric structures were obtained. The possible four different configurations (I-IV) of dimeric forms of the dipeptide were given in Fig. 1(d, e, f, g), respectively, together with their total energies. The geometric parameters of Dimer I are given in Table 1(b), in comparison to those of global conformation of mono Ala-Gln. The intra and inter hydrogen bonds, of the dimeric forms (I-IV) of the four low energy conformations, were given in Table 2. The number of inter H-bonds in Dimer $I$ is the highest, in comparison to those of the II-IV dimers. Thus the results indicate that inter hydrogen bonding interaction plays important role in determining the stable dimers. The intra H-bonds of mono Ala-Gln and inter and intra H-bonds of Dimer I are shown in Fig. 1(b, c), respectively.

For the amide plane five bond lengths and six bond angles considered and listed in Table 1(b), those are $4 \mathrm{C}-10 \mathrm{C}, 10 \mathrm{C}-11 \mathrm{O}, 10 \mathrm{C}-12 \mathrm{~N}, 12 \mathrm{~N}-14 \mathrm{C}, 12 \mathrm{~N}-13 \mathrm{H}, \angle \mathrm{CCO}(4,10,11), \angle \mathrm{CCN}(4,10,12), \angle \mathrm{OCN}(11$, $10,12), \angle \mathrm{CNC}(10,12,14), \angle \mathrm{CNH}(10,12,13)$ and $\angle \mathrm{HNC}(13,12,14)$, as indicated in Fig. 1(a). These five bond lengths and six bond angles are found to be in agreement with those of valine and phenylalanine containing dipeptides [5,7]. The geometry around alpha-carbon atom plays very important role in the structure of proteins. Ideally bond angle around carbon atom is $109.5^{\circ}$. But due to the streogenic nature of $\mathrm{C}_{\alpha}$ atom the ideal nature is not expected. For mono Ala-Gln unit, $\angle \mathrm{CC}_{\alpha}$ plane $(2 \mathrm{~N}, 4 \mathrm{C}, 10 \mathrm{C})$ is predicted as $111.17^{\circ}$, and for Dimer I, the corresponding angles $(2 \mathrm{~N}, 4 \mathrm{C}, 10 \mathrm{C}$ and $35 \mathrm{~N}, 33 \mathrm{C}, 32 \mathrm{C})$ are $111.47^{\circ}$ and $111.45^{\circ}$, respectively. The $\angle \mathrm{CC}_{\alpha}$ planes were found in $111.9^{\circ}-115.0^{\circ}$ ranges for valine containing dipeptides [5]. Thus our calculated results are compatible with those of previous findings $[5,7]$.

The dihedral angle of the dipeptide bond can give some information about the planarity of the peptide molecules, it should be $180^{\circ}$ if the amide plane is planar. The dihedral angles between $(4 \mathrm{C}, 10 \mathrm{C}, 12 \mathrm{~N}$, 14C) atoms (peptide bond: $10 \mathrm{C}-12 \mathrm{~N}$ ) of mono and Dimer I structures are $-174.40^{\circ}$ and $-179.40^{\circ}$, respectively. The other dihedral angle between (33C, 32C, 34N, 39C) atoms (peptide bond: 32C-34N) of Dimer I structure is $-179.5^{\circ}$. The results indicate that in Dimer I structure, the deviation of the peptide plane from planar structure is less than that of the monomer structure, probably due to the fact that inter hydrogen bonding interactions involving the $\mathrm{O}$ atoms on the amide planes forced the amide 


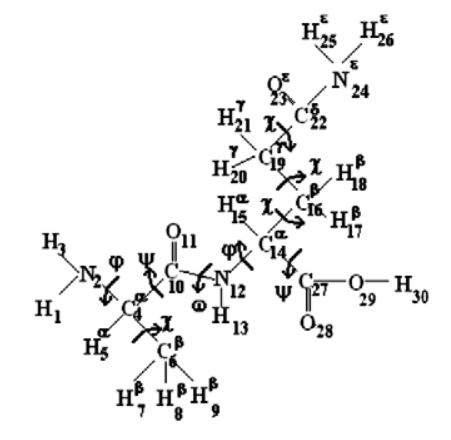

(a)

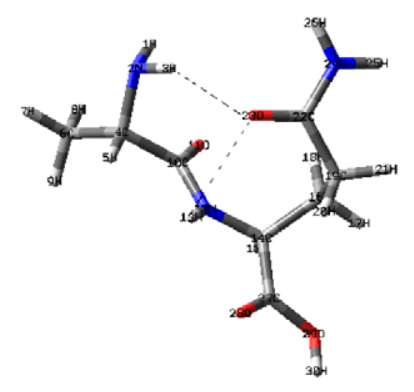

(b)

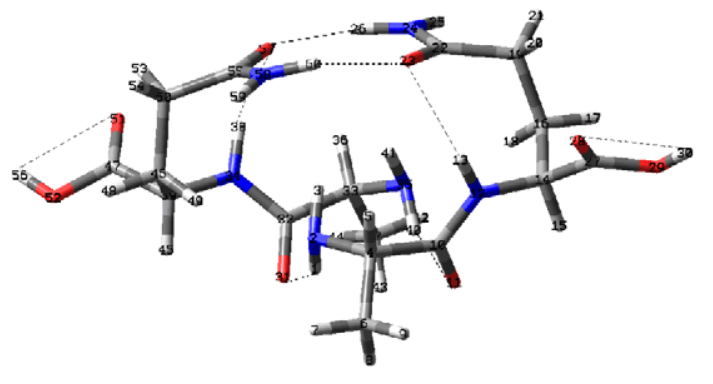

(c)

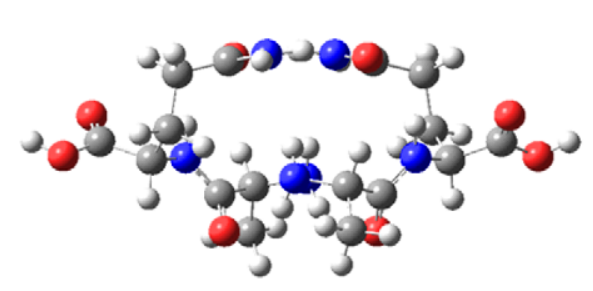

(d)

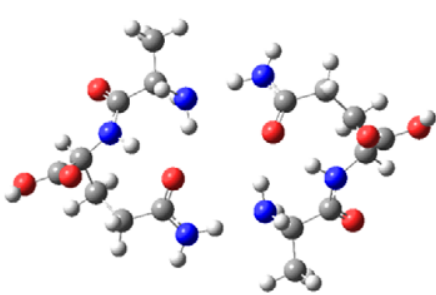

(e)

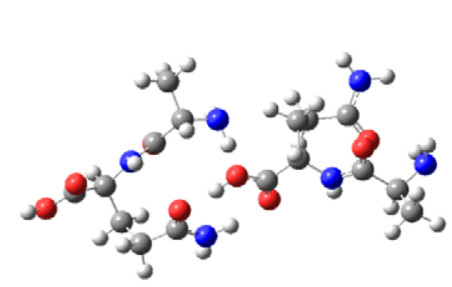

(f)

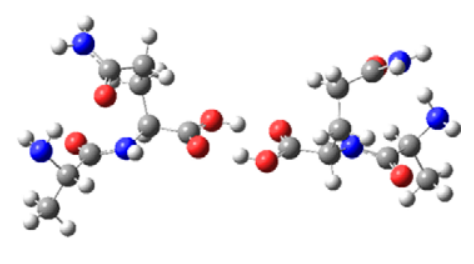

(g)

Fig. 1. The neutral form of Ala-Gln with the atom numbering and the dihedral angles used in Table 1 (a), the intra H-bonds of monomer Ala-Gln (b), inter and intra H-bonds of Dimer I (c), the geometrical structures of four low energy dimeric forms of Ala-Gln the energies obtained by DFT/B3LYP/6-31G(d,p) level of theory are $E=-977,809.95496405 \mathrm{kcal} / \mathrm{mol}$ Dimer I (d), $E=-977,809.60516250 \mathrm{kcal} / \mathrm{mol}$ Dimer II (e), $E=-977,809.52818043 \mathrm{kcal} / \mathrm{mol}$ Dimer III (f), $E=-977,809.08389576 \mathrm{kcal} / \mathrm{mol}$ Dimer IV (g). 
Table 1(a)

The torsional angles for the global conformation of the neutral Ala-Gln

\begin{tabular}{lccccccccc}
\hline Angles & $\mathrm{PHI}_{1}$ & $\mathrm{CH}_{11}$ & $\mathrm{PSI}_{1}$ & $\mathrm{~W}_{2}$ & $\mathrm{PHI}_{2}$ & $\mathrm{CH}_{21}$ & $\mathrm{CH}_{22}$ & $\mathrm{CH}_{23}$ & $\mathrm{PSI}_{2}$ \\
\hline Initial values & 50.00 & -60.00 & 60.00 & 180.00 & -150.00 & 60.00 & -60.00 & 90.00 & 150.00 \\
Optimized values & 50.27 & -60.28 & 71.74 & 180.87 & -150.31 & 58.25 & -76.21 & 101.43 & 156.78 \\
\hline
\end{tabular}

Table 1(b)

Geometry of mono Ala-Gln and Dimer I, calculated at the DFT level of theory using the standart 6-31G(d,p) basis set (distance in $\AA$, angles in degrees)

\begin{tabular}{|c|c|c|c|c|c|c|c|c|c|c|c|}
\hline Bond & Iono & Dimer I & Bond & Mono & Dimer I & Angle & Mono & Dimer I & Dihedral & Mono & Dimer I \\
\hline$\overline{\mathrm{H}_{1}-\mathrm{N}_{2}}$ & 1.0197 & 1.0212 & $\mathrm{O}_{29}-\mathrm{H}_{30}$ & 0.9725 & 0.9725 & $\mathrm{~N}_{2}-\mathrm{C}_{4}-\mathrm{C}_{6}$ & 110.00 & & $\mathrm{H}_{1}-\mathrm{N}_{2}-\mathrm{C}_{4}-\mathrm{C}_{6}$ & & \\
\hline${ }_{3}-\mathrm{N}_{2}$ & 1.0202 & 1.0174 & $\mathrm{~N}_{12}-\mathrm{C}_{14}$ & 1.4474 & 1.4475 & $\mathrm{~N}_{2}-\mathrm{C}_{4}-\mathrm{C}_{10}$ & 111.17 & 7 & $\mathrm{H}_{3}-\mathrm{N}_{2}-\mathrm{C}_{4}-\mathrm{C}_{6}$ & 174.90 & 174.96 \\
\hline $4-\mathrm{N}_{2}$ & 1.4680 & 1.4686 & $\mathrm{C}_{14}-\mathrm{H}_{15}$ & 1.0949 & 1.0 & $\mathrm{C}_{4}-\mathrm{C}_{10}-\mathrm{O}_{11}$ & 121.74 & 12 & $\mathrm{H}_{1}-\mathrm{N}_{2}-\mathrm{C}_{4}-\mathrm{C}_{10}$ & 9 & 56.88 \\
\hline$-\mathrm{C}_{4}$ & 1.5305 & 1.529 & $\mathrm{C}_{14}-\mathrm{C}_{16}$ & 9 & & $\mathrm{C}_{4}-\mathrm{C}_{10}-\mathrm{N}_{12}$ & 116 & & $\mathrm{~N}_{2}-\mathrm{C}_{4}-\mathrm{C}_{6}-\mathrm{H}_{7}$ & -5 & -53.50 \\
\hline$-\mathrm{H}_{7}$ & 1.0926 & 27 & $\mathrm{C}_{16}-\mathrm{H}_{17}$ & 33 & 4 & $\mathrm{O}_{11}-\mathrm{C}_{10}-\mathrm{N}_{12}$ & 7 & 61 & $\mathrm{C}_{4}-\mathrm{C}_{10}-\mathrm{N}_{12}-\mathrm{C}_{14}$ & 40 & -17 \\
\hline $6-\mathrm{H}_{8}$ & 1.0926 & 0927 & $\mathrm{C}_{16}-\mathrm{H}_{18}$ & 1.0933 & 1.0934 & $\mathbf{C}_{10}-\mathbf{N}_{12}-\mathbf{H}_{13}$ & 119 & 120.23 & $\mathrm{C}_{10}-\mathrm{N}_{12}-\mathrm{C}_{14}-\mathrm{C}_{27}$ & 16 & -13 \\
\hline $6-\mathrm{H}_{9}$ & 1.0926 & & $\mathrm{C}_{16}-\mathrm{C}_{19}$ & & & $\mathrm{C}_{10}-\mathrm{N}_{12}-\mathrm{C}_{14}$ & & & $\mathrm{C}_{10}-\mathrm{N}_{12}-\mathrm{C}_{14}-\mathrm{C}_{16}$ & & 15 \\
\hline${ }_{4}-C_{10}$ & 1.5377 & 1.5396 & $\mathrm{C}_{19}-\mathrm{H}_{20}$ & 33 & 1.0938 & $\mathrm{~N}_{12}-\mathrm{C}_{14}-\mathrm{C}_{16}$ & 114.55 & 11 & $\mathrm{~N}_{12}-\mathrm{C}_{14}-\mathrm{C}_{16}-\mathrm{C}_{19}$ & 9 & 59.06 \\
\hline${ }_{10}-\mathrm{O}_{11}$ & 1.23 & 26 & $\mathrm{C}_{19}-\mathrm{H}_{21}$ & 7 & & $\mathrm{H}_{13}-\mathrm{N}_{12}-\mathrm{C}_{14}$ & 110 & & $\mathrm{~N}_{2}-\mathrm{C}_{4}-\mathrm{C}_{10}-\mathrm{N}_{12}$ & & 3.07 \\
\hline$-\mathbf{N}_{12}$ & 1.3635 & 1.3620 & $\mathrm{C}_{19}-\mathrm{C}_{22}$ & 69 & 1.5 & $\mathrm{C}_{14}-\mathrm{C}_{16}-\mathrm{C}_{19}$ & 115 & & $\mathrm{H}_{13}-\mathrm{N}_{12}$ & 16 & 171.62 \\
\hline${ }_{2}-\mathbf{H}_{13}$ & 1.0114 & 1.0119 & $\mathrm{C}_{22}-\mathrm{O}_{23}$ & 73 & 1.2 & $\mathrm{C}_{16}-\mathrm{C}_{19}-\mathrm{C}_{22}$ & 113.33 & & $\mathrm{C}_{16}-\mathrm{C}_{19}-\mathrm{C}_{22}-\mathrm{N}_{24}$ & -87.43 & -87.31 \\
\hline & 1.0069 & 1.0081 & $\mathrm{C}_{22}-\mathrm{N}_{24}$ & 37 & 1.3 & $\mathrm{C}_{19}-\mathrm{C}_{22}-\mathrm{C}_{23}$ & 123.11 & 121.63 & $\mathrm{C}_{19}-\mathrm{C}_{22}-\mathrm{N}_{24}-\mathrm{H}_{25}$ & 5 & 5.0 \\
\hline $4-\mathrm{H}_{26}$ & 1.0089 & 1.0275 & & & & $\mathrm{C}_{19}-\mathrm{C}_{22}-\mathrm{N}_{24}$ & 11 & 11 & $\mathrm{~N}_{24}-\mathrm{H}_{26}$ & 174.41 & 171.70 \\
\hline$-\mathrm{C}_{27}$ & 1.5198 & 1.5231 & & & & $\mathrm{O}_{23}-\mathrm{C}_{22}-\mathrm{N}_{24}$ & 121 & 122 & $\mathrm{C}_{27}-\mathrm{O}_{29}$ & -70 & -72.43 \\
\hline$-\mathrm{O}_{28}$ & 1.2122 & 1.2114 & & & & $\mathrm{C}_{22}-\mathrm{N}_{24}-\mathrm{H}_{25}$ & & & $\mathrm{C}_{27}-\mathrm{O}_{28}$ & 49 & 104.9 \\
\hline $27-\mathrm{O}_{29}$ & 1.3503 & 1.3519 & & & & $\mathrm{C}_{22}-\mathrm{N}_{24}-\mathrm{H}_{26}$ & 118.28 & 120.19 & $\mathrm{~N}_{12}-\mathrm{C}_{14}-\mathrm{C}_{27}-\mathrm{O}_{29}$ & 163.81 & 161.3 \\
\hline
\end{tabular}

Table 2

The intra and inter hydrogen bonds of the dimeric forms (I-IV) of the four low energy conformations

\begin{tabular}{|c|c|c|c|c|c|c|c|}
\hline Atoms & Bond $(\AA)$ & Atoms & Bond $(\AA)$ & Atoms & Bond $(\AA)$ & Atoms & Bond $(\AA)$ \\
\hline \multicolumn{2}{|c|}{ Dimer I Intra-molecular } & \multicolumn{2}{|c|}{ Dimer II Intra-molecular } & \multicolumn{2}{|c|}{ Dimer III Intra-molecular } & \multicolumn{2}{|c|}{ Dimer IV Intra-molecular } \\
\hline \multicolumn{2}{|c|}{ H-bonds } & \multicolumn{2}{|c|}{ H-bonds } & \multicolumn{2}{|c|}{ H-bonds } & \multicolumn{2}{|c|}{ H-bonds } \\
\hline $510 \ldots 56 \mathrm{H}$ & 2.28917 & $13 \mathrm{H} \ldots .23 \mathrm{O}$ & 1.98321 & $41 \mathrm{H} \ldots .57 \mathrm{O}$ & 2.24862 & $3 \mathrm{H} . .23 \mathrm{O}$ & 2.24913 \\
\hline $28 \mathrm{O} \ldots 30 \mathrm{H}$ & 2.28921 & $38 \mathrm{H} \ldots 57 \mathrm{O}$ & 1.98469 & $13 \mathrm{H} . .28 \mathrm{O}$ & 2.25369 & $41 \mathrm{H} \ldots .57 \mathrm{O}$ & 2.24972 \\
\hline $13 \mathrm{H} \ldots .23 \mathrm{O}$ & 2.37558 & $41 \mathrm{H} \ldots .57 \mathrm{O}$ & 2.14085 & $38 \mathrm{H} \ldots .51 \mathrm{O}$ & 2.28370 & $38 \mathrm{H} \ldots .51 \mathrm{O}$ & 2.33653 \\
\hline \multirow[t]{5}{*}{$38 \mathrm{H} \ldots .57 \mathrm{O}$} & 2.38039 & $3 \mathrm{H} \ldots 23 \mathrm{O}$ & 2.14085 & $1 \mathrm{H} . .11 \mathrm{O}$ & 2.36494 & $13 \mathrm{H} \ldots .28 \mathrm{O}$ & 2.34236 \\
\hline & & $28 \mathrm{O} \ldots 30 \mathrm{H}$ & 2.28584 & $31 \mathrm{O} \ldots 40 \mathrm{H}$ & 2.61083 & $23 \mathrm{O} \ldots 26 \mathrm{H}$ & 2.49819 \\
\hline & & $51 \mathrm{O} \ldots 56 \mathrm{H}$ & 2.28585 & & & $57 \mathrm{O} \ldots 60 \mathrm{H}$ & 2.49892 \\
\hline & & & & & & $31 \mathrm{O} \ldots 40 \mathrm{H}$ & 2.72488 \\
\hline & & & & & & $1 \mathrm{H} . .11 \mathrm{O}$ & 2.73040 \\
\hline \multicolumn{2}{|c|}{ Inter-molecular } & \multicolumn{2}{|c|}{ Inter-molecular } & \multicolumn{2}{|c|}{ Inter-molecular } & \multicolumn{2}{|c|}{ Inter-molecular } \\
\hline \multicolumn{2}{|c|}{ H-bonds } & \multicolumn{2}{|c|}{ H-bonds } & \multicolumn{2}{|c|}{ H-bonds } & \multicolumn{2}{|c|}{ H-bonds } \\
\hline $23 \mathrm{O} \ldots 60 \mathrm{H}$ & 1.87079 & $35 \mathrm{~N} \ldots 26 \mathrm{H}$ & 1.89284 & $23 \mathrm{O} \ldots 56 \mathrm{H}$ & 1.60939 & $28 \mathrm{O} \ldots .56 \mathrm{H}$ & 1.63684 \\
\hline $26 \mathrm{H} \ldots .57 \mathrm{O}$ & 1.87155 & $2 \mathrm{~N} \ldots 60 \mathrm{H}$ & 1.89293 & $26 \mathrm{H} \ldots .51 \mathrm{O}$ & 1.88003 & $30 \mathrm{H} \ldots .51 \mathrm{O}$ & 1.63825 \\
\hline $11 \mathrm{O} \ldots 40 \mathrm{H}$ & 2.33151 & & & & & & \\
\hline $1 \mathrm{H} . .31 \mathrm{O}$ & 2.33838 & & & & & & \\
\hline
\end{tabular}




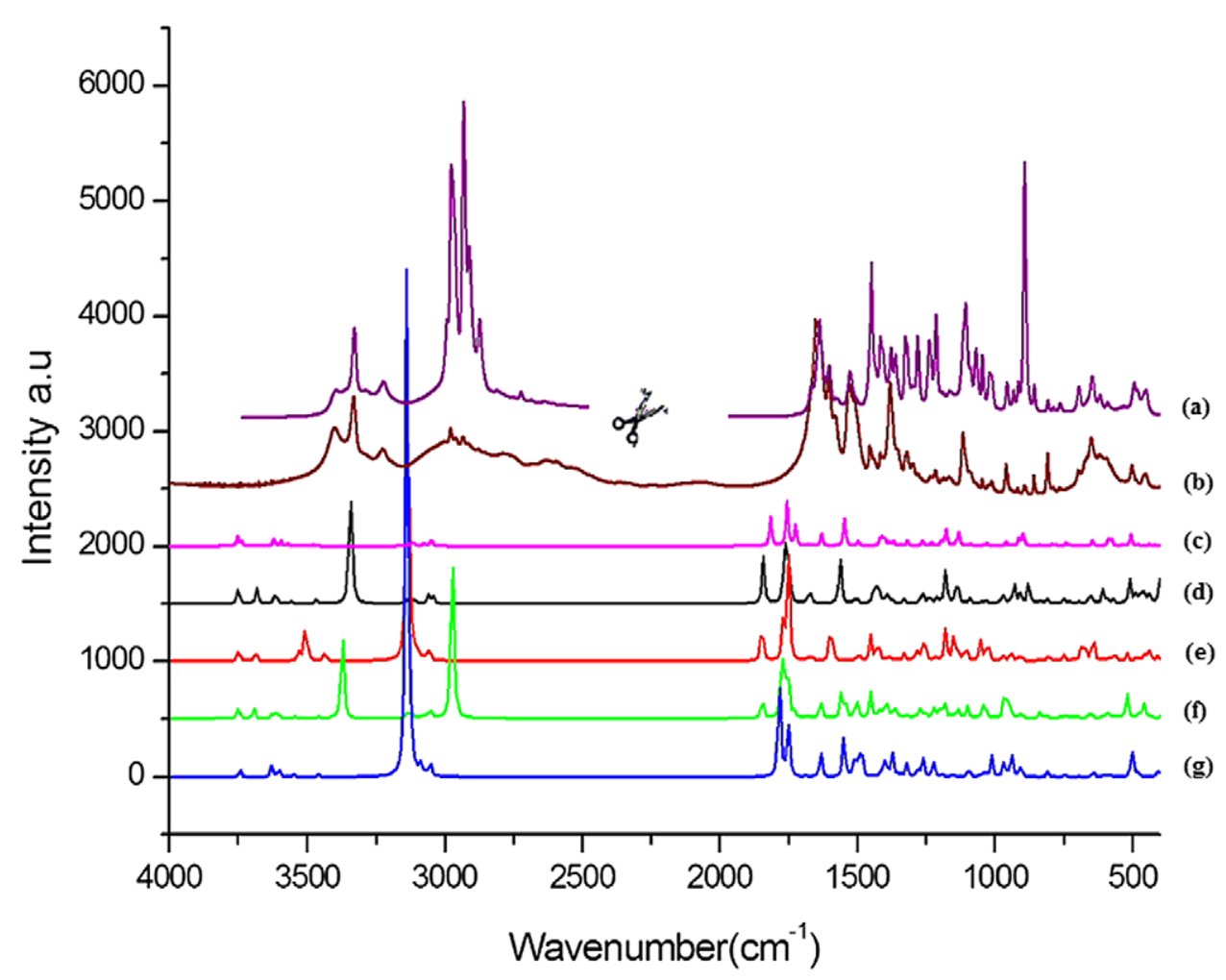

Fig. 2. The experimental micro Raman (a) and FT-IR spectra (b) of Ala-Gln dipeptide, and calculated IR spectra of monomeric (c) and dimeric forms (I-IV) the dipeptide (d-g); Dimer I (d), Dimer II (e), Dimer III (f) Dimer IV (g).

planes of Dimer I structure to be more planar structure; in the Dimer I structure, one of the hydrogens of the N-terminal group of Alanine involves hydrogen bonding interaction with the oxygen atom of the $\mathrm{C}=\mathrm{O}$ group of Alanine of the other pair $(1 \mathrm{H} \ldots 31 \mathrm{O})$, on the other hand the $\mathrm{N}$-terminal group of Alanine molecule of the other pair involves hydrogen bonding interaction with the oxygen atom of the $\mathrm{C}=\mathrm{O}$ group of Alanine of the first pair (40H...11O) (see Table 2).

The calculated wavenumbers and the total energy distribution of the vibrational modes of mono AlaGln dipeptide are given in Table 3, in comparison with the experimental IR and Raman spectra of the investigated dipeptide and the calculated wavenumbers of Dimer I. On the other hand, the calculated vibrational wavenumbers of Ala-Gln dimers (I-IV) were tabulated in Table 4. According to the frequency data in Tables 3 and 4, no imaginary frequency has been observed at the optimized structures of the title molecule and its dimers, proving that a true minima on the potential surfaces were found. The experimental micro Raman and FT-IR spectra of Ala-Gln, in comparison with the calculated IR spectrum of global conformation of mono Ala-Gln and Dimer I units are given in Fig. 2(a, b, c, d), respectively. The calculated IR spectra of low energy conformers of Ala-Gln dimmers (Dimers I-IV) are given in Fig. 2(d, $\mathrm{e}, \mathrm{f}, \mathrm{g})$, respectively.

The C-H stretching vibrations of alifatic structures often occur in the region of $2936-2843 \mathrm{~cm}^{-1}$ [8]. In our work, the weak band observed at $2965 \mathrm{~cm}^{-1}$ in IR spectra and the very strong band at $2932 \mathrm{~cm}^{-1}$ in Raman spectra have been assigned as methylene group of glutamine asymmetric and symmetric stretching, respectively. In theoretical calculations, these stretching modes are predicted at $2969 \mathrm{~cm}^{-1}$ \{with 6-31G $\left.++(\mathrm{d}, \mathrm{p})\right\}$ and $2931 \mathrm{~cm}^{-1}$ \{with 6-31G $\left.++(\mathrm{d}, \mathrm{p})\right\}$, respectively after scaling with 
Table 3

Calculated and experimental wavenumbes $\left(\mathrm{cm}^{-1}\right)$ and the total energy distribution of the vibrational modes of the Ala-Gln and calculated wavenumbers of Dimer I

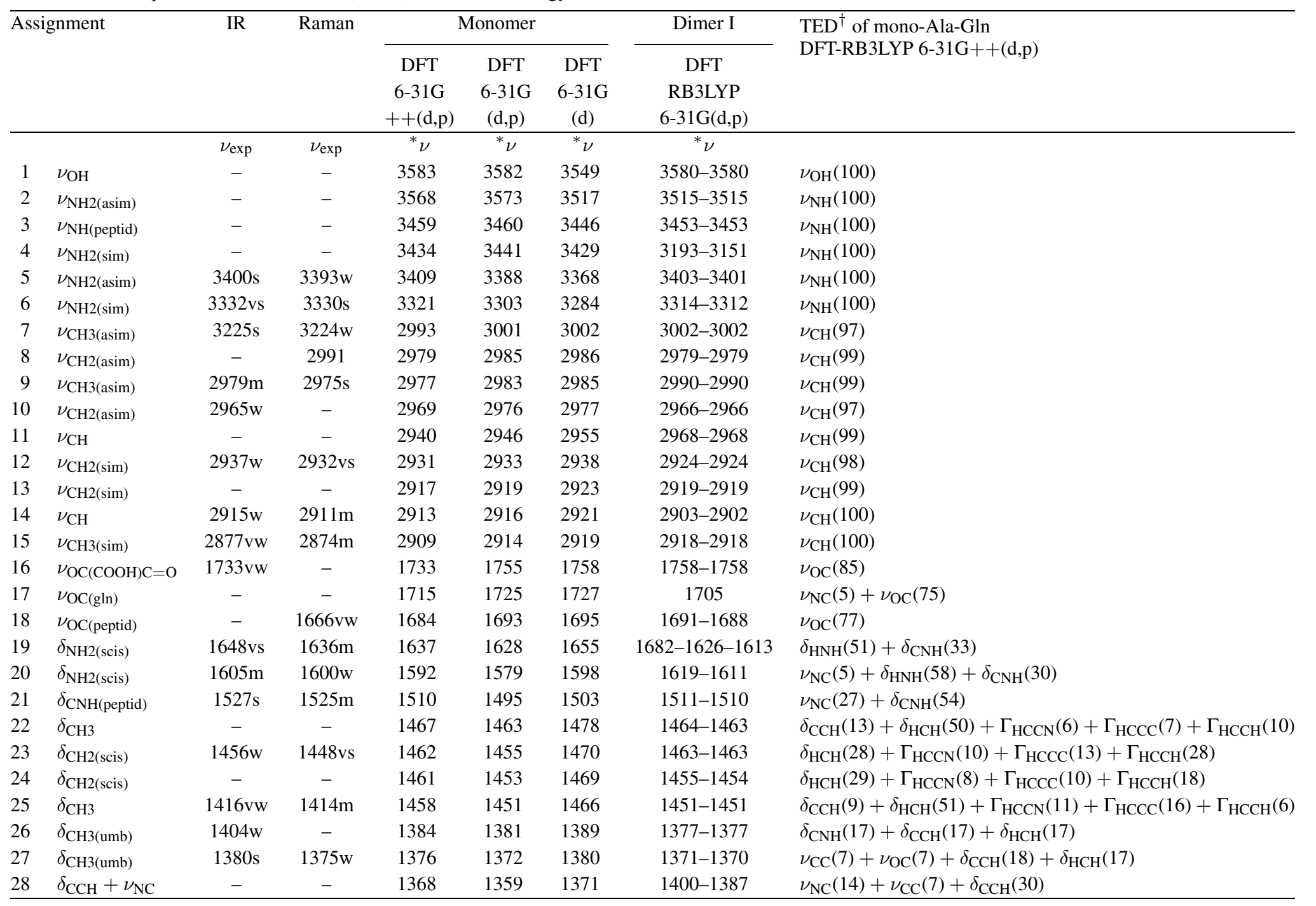


Table 3

(Continued)

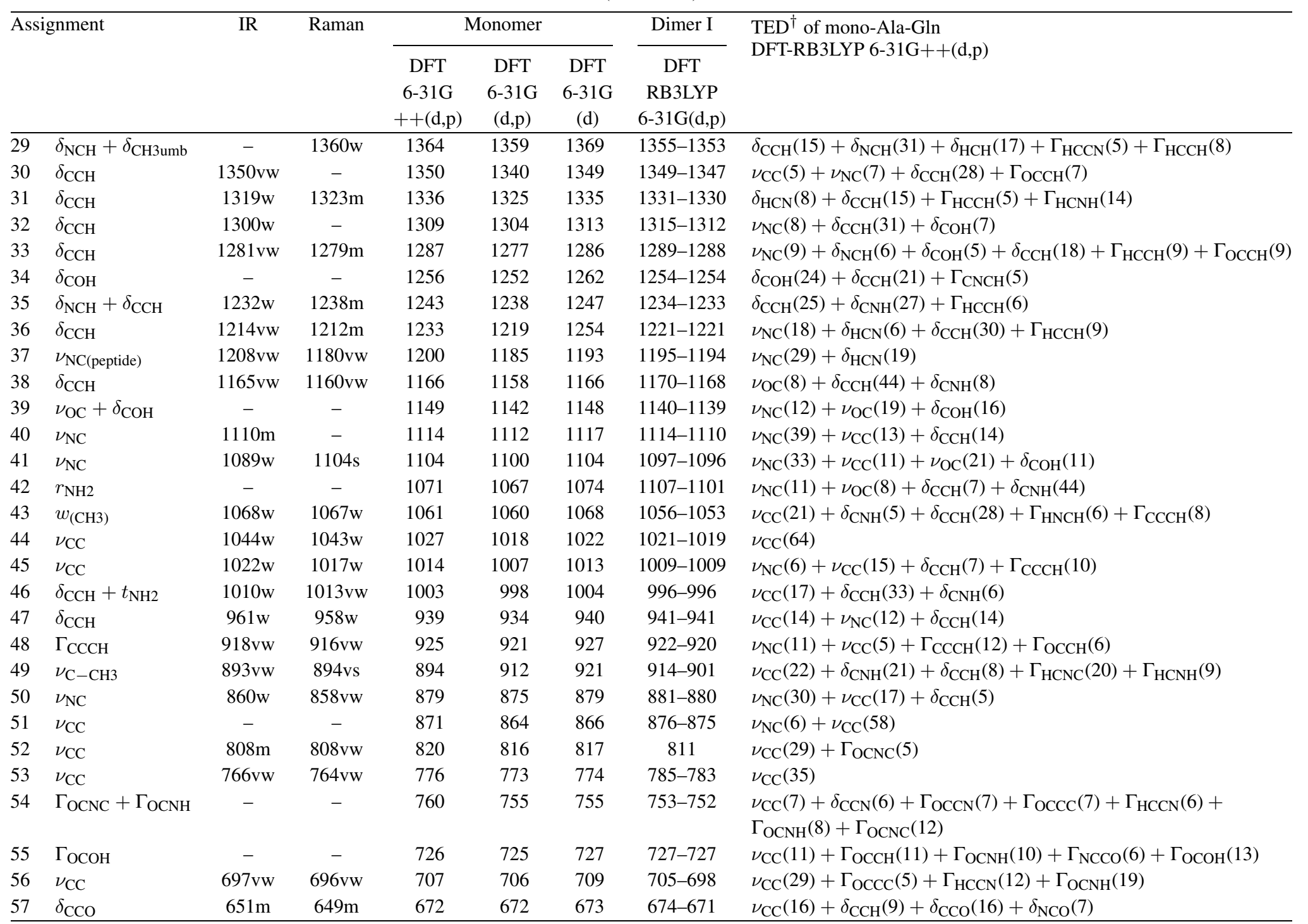


Table 3

(Continued)

\begin{tabular}{|c|c|c|c|c|c|c|c|c|}
\hline & \multirow[t]{4}{*}{ IR } & \multirow[t]{4}{*}{ Raman } & \multicolumn{3}{|c|}{ Monomer } & \multirow{4}{*}{$\begin{array}{c}\text { Dimer I } \\
\text { DFT } \\
\text { RB3LYP } \\
6-31 G(d, p)\end{array}$} & \multirow{4}{*}{$\begin{array}{l}\mathrm{TED}^{\dagger} \text { of mono-Ala-Gln } \\
\text { DFT-RB3LYP 6-31G++(d,p) }\end{array}$} \\
\hline \multirow{3}{*}{\multicolumn{2}{|c|}{ Assignment }} & & & DFT & DFT & DFT & & \\
\hline & & & & $6-31 \mathrm{G}$ & $6-31 G$ & $6-31 \mathrm{G}$ & & \\
\hline & & & & $++(\mathrm{d}, \mathrm{p})$ & $(\mathrm{d}, \mathrm{p})$ & (d) & & \\
\hline$\overline{58}$ & $\Gamma_{\mathrm{CCOH}}$ & $620 \mathrm{vw}$ & $619 \mathrm{vw}$ & 632 & 640 & 643 & $633-632$ & $\delta_{\mathrm{CCO}}(8)+\Gamma_{\mathrm{HOCO}}(27)+\Gamma_{\mathrm{HOCC}}(18)$ \\
\hline 59 & $\Gamma_{\mathrm{CCOH}}$ & $596 \mathrm{vw}$ & $592 \mathrm{vw}$ & 576 & 580 & 583 & $588-587$ & $\nu_{\mathrm{CC}}(9)+\delta_{\mathrm{CCO}}(10)+\delta_{\mathrm{OCO}}(14)+\Gamma_{\mathrm{HOCO}}(7)+\Gamma_{\mathrm{HOCC}}(19)$ \\
\hline 60 & $t_{(\mathrm{NH} 2)}+\Gamma_{\mathrm{CCNH}}$ & - & - & 565 & 562 & 563 & $849-816$ & $\delta_{\mathrm{CCO}}(6)+\delta_{\mathrm{OCO}}(14)+\Gamma_{\mathrm{CCNH}}(22)+\Gamma_{\mathrm{OCNH}}(13)$ \\
\hline 61 & $\Gamma_{\mathrm{CCNH}}+\Gamma_{\mathrm{HOCC}}$ & - & - & 562 & 558 & 562 & $551-547$ & $\delta_{\mathrm{CCO}}(9)+\delta_{\mathrm{NCO}}(8)+\Gamma_{\mathrm{CCNH}}(16)+\Gamma_{\mathrm{OCNH}}(11)+\Gamma_{\mathrm{HOCC}}(11)$ \\
\hline 62 & $\delta_{\mathrm{NCO}}$ & $532 \mathrm{vw}$ & $531 \mathrm{vw}$ & 527 & 524 & 525 & $570-566$ & $\nu_{\mathrm{CC}}(10)+\delta_{\mathrm{CCO}}(16)+\delta_{\mathrm{NCO}}(33)$ \\
\hline 63 & $\Gamma_{\mathrm{CCNH}}$ & $503 \mathrm{w}$ & $495 w$ & 496 & 492 & 495 & $499-494-492$ & $\Gamma_{\mathrm{OCNH}}(26)+\Gamma_{\mathrm{CCNH}}(47)$ \\
\hline 64 & $\delta_{\mathrm{NCC}}$ & $454 w$ & $452 w$ & 467 & 461 & 462 & $464-451-442$ & $\delta_{\mathrm{NCC}}(18)+\delta_{\mathrm{CCC}}(7)+\Gamma_{\mathrm{OCCC}}(7)$ \\
\hline 65 & $\delta_{\mathrm{CCO}}+t_{(\mathrm{NH} 2)}$ & $422 \mathrm{vw}$ & - & 429 & 424 & 424 & 427 & $\delta_{\mathrm{NCC}}(10)+\delta_{\mathrm{CCO}}(25)+\Gamma_{\mathrm{CCNH}}(11)$ \\
\hline 66 & $\delta_{\mathrm{NCC}}$ & $419 \mathrm{vw}$ & - & 409 & 407 & 407 & $422-414$ & $\delta_{\mathrm{CCO}}(27)+\delta_{\mathrm{NCC}}(46)$ \\
\hline 67 & $r_{(\mathrm{NH} 2)}$ & - & $359 \mathrm{vw}$ & 365 & 380 & 381 & $388-337$ & $\Gamma_{\mathrm{HNCH}}(16)+\Gamma_{\mathrm{HNCC}}(57)$ \\
\hline 68 & $\Gamma_{\mathrm{CCNH}}$ & - & $350 w$ & 324 & 319 & 320 & $345-339$ & $\nu_{\mathrm{CC}}(10)+\delta_{\mathrm{NCC}}(14)+\delta_{\mathrm{CCC}}(12)+\Gamma_{\mathrm{HNCC}}(20)$ \\
\hline 69 & $\delta_{\mathrm{NCC}}$ & - & $291 w$ & 298 & 301 & 301 & $312-304$ & $\nu_{\mathrm{CC}}(10)+\delta_{\mathrm{NCO}}(8)+\delta_{\mathrm{NCC}}(18)+\delta_{\mathrm{CCC}}(7)+\delta_{\mathrm{OCC}}(11)+\Gamma_{\mathrm{HNCC}}(15)$ \\
\hline 70 & $\delta_{\mathrm{CCC}}$ & - & - & 280 & 280 & 280 & $293-291$ & $\delta_{\mathrm{CCC}}(27)+\Gamma_{\mathrm{HNCC}}(15)+\Gamma_{\mathrm{CNCO}}(8)+\Gamma_{\mathrm{HNCO}}(6)$ \\
\hline 71 & $\delta_{\mathrm{CCC}}$ & - & - & 269 & 270 & 269 & $271-270$ & $\delta_{\mathrm{NCC}}(16)+\delta_{\mathrm{CCC}}(27)+\Gamma_{\mathrm{HNCC}}(20)$ \\
\hline 72 & $t_{(\mathrm{NH} 2)}+\Gamma_{\mathrm{CCNH}}$ & - & - & 255 & 248 & 247 & 475 & $\Gamma_{\mathrm{HNCC}}(41)+\Gamma_{\mathrm{HNCO}}(39)$ \\
\hline 73 & $\Gamma_{\mathrm{CCNH}}$ & - & $248 \mathrm{vw}$ & 244 & 239 & 229 & $248-247$ & $\nu_{\mathrm{CC}}(7)+\delta_{\mathrm{NCC}}(16)+\delta_{\mathrm{CCC}}(13)+\Gamma_{\mathrm{HNCC}}(33)+\Gamma_{\mathrm{HCCC}}(7)$ \\
\hline 74 & $\Gamma_{\mathrm{HCCC}}$ & - & $229 w$ & 223 & 224 & 223 & $224-222$ & $\Gamma_{\mathrm{HCCH}}(16)+\Gamma_{\mathrm{HCCC}}(56)$ \\
\hline 75 & $\delta_{\mathrm{CCC}}$ & - & - & 205 & 205 & 204 & $216-215$ & $\delta_{\mathrm{NCC}}(14)+\delta_{\mathrm{CCC}}(29)+\Gamma_{\mathrm{OCCH}}(5)+\Gamma_{\mathrm{NCCO}}(10)$ \\
\hline 76 & $\delta_{\mathrm{CCC}}$ & - & - & 148 & 149 & 151 & $176-172$ & $\delta_{\mathrm{CCC}}(41)+\Gamma_{\mathrm{CNCC}}(5)+\Gamma_{\mathrm{HNCC}}(5)$ \\
\hline 77 & $\delta_{\mathrm{CNC}}$ & - & - & 114 & 117 & 118 & $152-150$ & $\delta_{\mathrm{NCC}}(34)+\delta_{\mathrm{CNH}}(8)+\delta_{\mathrm{CCC}}(5)+\Gamma_{\mathrm{HCCC}}(10)$ \\
\hline 78 & $\Gamma_{\mathrm{NCCC}}$ & - & - & 105 & 115 & 116 & 135 & $\delta_{\mathrm{NCC}}(7)+\Gamma_{\mathrm{HNCC}}(20)+\Gamma_{\mathrm{NCCC}}(24)+\Gamma_{\mathrm{HCCC}}(17)+\Gamma_{\mathrm{CCCC}}(15)$ \\
\hline 79 & $\Gamma_{\mathrm{NCCC}}$ & - & - & 75 & 89 & 87 & $103-83$ & $\Gamma_{\mathrm{NCCC}}(65)+\Gamma_{\mathrm{OCNC}}(10)+\Gamma_{\mathrm{CNCH}}(11)+\Gamma_{\mathrm{CCCO}}(7)$ \\
\hline 80 & $\Gamma_{\mathrm{CCNH}}$ & - & - & 60 & 65 & 66 & $71-65-40$ & $\Gamma_{\mathrm{HNCC}}(24)+\Gamma_{\mathrm{OCNH}}(6)+\Gamma_{\mathrm{CNCC}}(21)+\Gamma_{\mathrm{CCCC}}(7)+\Gamma_{\mathrm{OCCC}}(9)$ \\
\hline 81 & $\Gamma_{\mathrm{OCCN}}$ & - & - & 55 & 60 & 60 & $\begin{array}{c}88-87 \\
29-22-20\end{array}$ & $\Gamma_{\mathrm{OCCN}}(36)+\Gamma_{\mathrm{OCCH}}(15)+\Gamma_{\mathrm{OCCC}}(9)+\Gamma_{\mathrm{NCCN}}(10)+\Gamma_{\mathrm{NCCH}}(7)$ \\
\hline 82 & $\Gamma_{\mathrm{OCCC}}$ & - & - & 46 & 47 & 47 & $45-47$ & $\Gamma_{\mathrm{HCCC}}(7)+\Gamma_{\mathrm{OCCH}}(12)+\Gamma_{\mathrm{OCCC}}(52)$ \\
\hline 83 & $\Gamma_{\mathrm{CCCH}}$ & - & - & 26 & 27 & 28 & $55-33$ & $\Gamma_{\mathrm{NCCN}}(11)+\Gamma_{\mathrm{NCCC}}(15)+\Gamma_{\mathrm{NCCH}}(14)+\Gamma_{\mathrm{HCCC}}(30)+\Gamma_{\mathrm{CCCC}}(13)$ \\
\hline 84 & $\Gamma_{\mathrm{CCNH}}$ & - & - & 19 & 13 & 20 & $97-29$ & $\Gamma_{\mathrm{HNCC}}(30)+\Gamma_{\mathrm{HNCH}}(7)+\Gamma_{\mathrm{CNCC}}(27)+\Gamma_{\mathrm{CCCH}}(5)+\Gamma_{\mathrm{OCCC}}(7)$ \\
\hline
\end{tabular}

Notes: ${ }^{\dagger}$ Only contributions $>5 \%$ are listed.

${ }^{*}$ The wavenumbers under $1800 \mathrm{~cm}^{-1}$ were scaled with 0.967 and 0.977 for DFT 6-31G(d,p) and DFT 6-31G++(d,p) respectively and for over $1800 \mathrm{~cm}^{-1}$ the scale factor 0.955 were used for both DFT 6-31G(d,p) and DFT 6-31G $++(\mathrm{d}, \mathrm{p})$ levels of theory. 
Table 4

Calculated (B3LYP/6-31G(d,p)) wavenumbers $\left(\mathrm{cm}^{-1}\right)$ for four conformers of Ala-Gln Dimers (I-IV)

\begin{tabular}{|c|c|c|c|c|c|c|c|c|c|c|c|c|c|c|c|}
\hline \multicolumn{2}{|c|}{ Assign. Dimer I } & \multirow{2}{*}{$\begin{array}{c}\text { IR } \\
\nu_{\exp }\end{array}$} & \multirow{2}{*}{$\frac{\text { Raman }}{\nu_{\text {exp }}}$} & \multirow{2}{*}{$\begin{array}{l}\mathrm{I} \\
\nu\end{array}$} & \multirow{2}{*}{$\begin{array}{l}\mathrm{II} \\
{ }^{*} \nu\end{array}$} & \multirow{2}{*}{$\begin{array}{l}\text { III } \\
{ }^{*} \nu\end{array}$} & \multirow{2}{*}{$\begin{array}{l}\mathrm{IV} \\
{ }^{*} \nu\end{array}$} & \multicolumn{2}{|c|}{ Assign. Dimer I } & \multirow{2}{*}{$\begin{array}{c}\mathrm{IR} \\
\nu_{\exp }\end{array}$} & \multirow{2}{*}{$\begin{array}{c}\text { Raman } \\
\nu_{\exp }\end{array}$} & \multirow{2}{*}{$\begin{array}{l}\mathrm{I} \\
{ }^{*} \nu\end{array}$} & \multirow{2}{*}{$\begin{array}{l}\text { II } \\
{ }^{*} \nu\end{array}$} & \multirow{2}{*}{$\begin{array}{l}\text { III } \\
{ }^{*} \nu\end{array}$} & \multirow{2}{*}{$\begin{array}{l}\mathrm{IV} \\
{ }^{*} \nu\end{array}$} \\
\hline & & & & & & & & & & & & & & & \\
\hline 1 & $\nu_{\mathrm{OH}}$ & & & 3580 & 3578 & 3582 & 3574 & 88 & $\nu_{\mathrm{CC}}$ & & & 1019 & 1029 & 1018 & 1020 \\
\hline 2 & $\nu_{\mathrm{OH}}$ & & & 3580 & 3578 & 3575 & 3573 & 89 & $\nu_{\mathrm{CC}}$ & $1022 w$ & $1017 \mathrm{w}$ & 1009 & 1022 & 1013 & 1011 \\
\hline 3 & $\nu_{\mathrm{NH} 2 \text { (asim) }}$ & & & 3515 & 3519 & 3526 & 3466 & 90 & $\nu_{\mathrm{CC}}$ & & & 1009 & 1016 & 1010 & 1011 \\
\hline 4 & $\nu_{\mathrm{NH} 2(\operatorname{asim})}$ & & & 3515 & 3519 & 3463 & 3466 & 91 & $\delta_{\mathrm{CCH}}+t_{(\mathrm{NH} 2)}$ & & & 996 & 1014 & 1003 & 1000 \\
\hline 5 & $\nu_{\mathrm{NH}(\text { peptid })}$ & & & 3453 & 3374 & 3452 & 3442 & 92 & $\delta_{\mathrm{CCH}}+t_{(\mathrm{NH} 2)}$ & $1010 w$ & $1013 \mathrm{vw}$ & 996 & 1011 & 998 & 1000 \\
\hline 6 & $\nu_{\mathrm{NH}(\text { peptid }}$ & & & 3453 & 3374 & 3442 & 3441 & 93 & $\delta_{\mathrm{CCH}}$ & & & 941 & 993 & 993 & 977 \\
\hline 7 & $\nu_{\mathrm{NH} 2(\operatorname{asim})}$ & & & 3403 & 3349 & 3387 & 3390 & 94 & $\delta_{\mathrm{CCH}}$ & $961 w$ & $958 w$ & 941 & 989 & 936 & 938 \\
\hline 8 & $\nu_{\mathrm{NH} 2 \text { (asim) }}$ & $3400 \mathrm{~s}$ & $3393 w$ & 3401 & 3348 & 3386 & 3389 & 95 & $\Gamma_{\mathrm{CCCH}}$ & $918 \mathrm{vw}$ & $916 \mathrm{vw}$ & 922 & 941 & 935 & 936 \\
\hline 9 & $\nu_{\mathrm{NH} 2(\operatorname{sim})}$ & $3332 \mathrm{vs}$ & $3330 \mathrm{~s}$ & 3314 & 3282 & 3311 & 3303 & 96 & $\Gamma_{\mathrm{CCCH}}$ & & & 920 & 939 & 925 & 936 \\
\hline 10 & $\nu_{\mathrm{NH} 2(\operatorname{sim})}$ & & & 3312 & 3281 & 3304 & 3303 & 97 & $\nu_{\mathrm{CC}-\mathrm{CH} 3}$ & $893 \mathrm{vw}$ & $894 v s$ & 914 & 914 & 923 & 921 \\
\hline 11 & $\nu_{\mathrm{NH} 2(\operatorname{sim})}$ & & & 3193 & 3000 & 3222 & 3003 & 98 & $\nu_{\mathrm{CC}-\mathrm{CH} 3}$ & & & 901 & 913 & 921 & 921 \\
\hline 12 & $\nu_{\mathrm{NH} 2(\operatorname{sim})}$ & & & 3151 & 3000 & 3000 & 3002 & 99 & $\nu_{\mathrm{NC}}$ & & & 881 & 911 & 915 & 909 \\
\hline 13 & $\nu_{\mathrm{CH} 3 \text { (asim) }}$ & & & 3002 & 2995 & 2999 & 2997 & 100 & $\nu_{\mathrm{NC}}$ & $860 w$ & $858 \mathrm{vw}$ & 880 & 910 & 882 & 909 \\
\hline 14 & $\nu_{\mathrm{CH} 3 \text { (asim) }}$ & & & 3002 & 2991 & 2992 & 2986 & 101 & $\nu_{\mathrm{CC}}$ & & & 876 & 884 & 882 & 882 \\
\hline 15 & $\nu_{\mathrm{CH} 3 \text { (asim) }}$ & & & 2990 & 2991 & 2985 & 2985 & 102 & $\nu_{\mathrm{CC}}$ & & & 875 & 884 & 873 & 881 \\
\hline 16 & $\nu_{\mathrm{CH} 3 \text { (asim) }}$ & $2979 m$ & $2975 \mathrm{~s}$ & 2990 & 2980 & 2984 & 2985 & 103 & $t_{(\mathrm{NH} 2)}+\Gamma_{\mathrm{CCNH}}$ & & & 849 & 872 & 872 & 869 \\
\hline 17 & $\nu_{\mathrm{CH} 2 \text { (asim) }}$ & & & 2979 & 2977 & 2982 & 2985 & 104 & $t_{(\mathrm{NH} 2)}$ & & & 816 & 872 & 827 & 869 \\
\hline 18 & $\nu_{\mathrm{CH} 2 \text { (asim) }}$ & & & 2979 & 2977 & 2980 & 2976 & 105 & $\nu_{\mathrm{CC}}$ & $808 \mathrm{~m}$ & $808 \mathrm{vw}$ & 811 & 809 & 814 & 829 \\
\hline 19 & $\nu_{\mathrm{CH}}$ & & & 2968 & 2967 & 2977 & 2976 & 106 & $t_{(\mathrm{NH} 2)}$ & & & 806 & 809 & 810 & 826 \\
\hline 20 & $\nu_{\mathrm{CH}}$ & & & 2968 & 2967 & 2977 & 2950 & 107 & $\nu_{\mathrm{CC}}$ & & & 785 & 779 & 788 & 783 \\
\hline 21 & $\nu_{\mathrm{CH} 2 \text { (asim) }}$ & & & 2966 & 2957 & 2952 & 2950 & 108 & $\nu_{\mathrm{CC}}$ & & & 783 & 779 & 784 & 779 \\
\hline 22 & $\nu_{\mathrm{CH} 2 \text { (asim) }}$ & $2965 w$ & & 2966 & 2957 & 2941 & 2936 & 109 & $\Gamma_{\text {OCNC }}+\Gamma_{\text {OCNHpeptide }}$ & $766 \mathrm{vw}$ & $764 \mathrm{vw}$ & 753 & 754 & 755 & 758 \\
\hline 23 & $\nu_{\mathrm{CH} 2(\operatorname{sim})}$ & & & 2924 & 2936 & 2935 & 2936 & 110 & $\Gamma_{\text {OCNC }}+\Gamma_{\text {OCNHpeptide }}$ & & & 752 & 753 & 741 & 757 \\
\hline 24 & $\nu_{\mathrm{CH} 2(\operatorname{sim})}$ & $2937 w$ & $2932 \mathrm{vs}$ & 2924 & 2936 & 2929 & 2920 & 111 & $\Gamma_{\mathrm{OCOH}}$ & & & 727 & 726 & 730 & 728 \\
\hline 25 & $\nu_{\mathrm{CH} 2(\operatorname{sim})}$ & & & 2919 & 2923 & 2925 & 2920 & 112 & $\Gamma_{\mathrm{OCOH}}$ & & & 727 & 726 & 719 & 722 \\
\hline 26 & $\nu_{\mathrm{CH} 2(\operatorname{sim})}$ & & & 2919 & 2923 & 2922 & 2916 & 113 & $\nu_{\mathrm{CC}}$ & & & 705 & 697 & 708 & 713 \\
\hline 27 & $\nu_{\mathrm{CH} 3(\operatorname{sim})}$ & $2877 \mathrm{vw}$ & $2874 \mathrm{~m}$ & 2918 & 2920 & 2917 & 2915 & 114 & $\nu_{\mathrm{CC}}$ & $697 \mathrm{vw}$ & $696 \mathrm{vw}$ & 698 & 697 & 692 & 711 \\
\hline 28 & $\nu_{\mathrm{CH} 3(\operatorname{sim})}$ & & & 2918 & 2920 & 2913 & 2913 & 115 & $\delta_{\mathrm{CCO}}$ & $651 \mathrm{~m}$ & $647 \mathrm{~m}$ & 674 & 668 & 683 & 674 \\
\hline 29 & $\nu_{\mathrm{CH}}$ & $2915 w$ & $2911 \mathrm{~m}$ & 2903 & 2914 & 2913 & 2913 & 116 & $\delta_{\mathrm{CCO}}$ & & & 671 & 666 & 676 & 672 \\
\hline 30 & $\nu_{\mathrm{CH}}$ & & & 2902 & 2914 & 2840 & 2885 & 117 & $\Gamma_{\mathrm{CCOH}}$ & & & 633 & 654 & 642 & 623 \\
\hline 31 & $\nu_{\mathrm{OC}(\mathrm{COOH}) \mathrm{C}=\mathrm{O}}$ & $1733 \mathrm{vw}$ & & 1758 & 1762 & 1761 & 1724 & 118 & $\Gamma_{\mathrm{CCOH}}$ & $620 \mathrm{vw}$ & $619 \mathrm{vw}$ & 632 & 651 & 632 & 617 \\
\hline 32 & $\nu_{\mathrm{OC}(\mathrm{COOH}) \mathrm{C}=\mathrm{O}}$ & & & 1758 & 1762 & 1718 & 1724 & 119 & $\Gamma_{\mathrm{CCOH}}$ & $596 \mathrm{vw}$ & $592 \mathrm{vw}$ & 588 & 623 & 591 & 590 \\
\hline 33 & $\nu_{\mathrm{OC}(\mathrm{gln})}$ & & & 1705 & 1711 & 1712 & 1720 & 120 & $\Gamma_{\mathrm{CCOH}}$ & & & 587 & 622 & 575 & 583 \\
\hline
\end{tabular}


Table 4

(Continued)

\begin{tabular}{|c|c|c|c|c|c|c|c|c|c|c|c|c|c|c|c|}
\hline \multicolumn{2}{|c|}{ Assign. Dimer I } & \multirow[t]{2}{*}{ IR } & \multirow[t]{2}{*}{ Raman } & \multirow{2}{*}{$\begin{array}{c}\mathrm{I} \\
1691\end{array}$} & \multirow{2}{*}{$\begin{array}{c}\text { II } \\
1710\end{array}$} & \multirow{2}{*}{$\begin{array}{c}\text { III } \\
1700\end{array}$} & \multirow{2}{*}{$\begin{array}{c}\text { IV } \\
1697\end{array}$} & \multicolumn{2}{|c|}{ Assign. Dimer I } & \multirow[t]{2}{*}{ IR } & \multirow[t]{2}{*}{ Raman } & \multirow{2}{*}{$\begin{array}{c}\mathrm{I} \\
570\end{array}$} & \multirow{2}{*}{$\begin{array}{c}\text { II } \\
585\end{array}$} & \multirow{2}{*}{$\begin{array}{l}\text { III } \\
567\end{array}$} & \multirow{2}{*}{$\frac{\mathrm{IV}}{566}$} \\
\hline 34 & $\nu_{\mathrm{OC}(\text { peptide })}$ & & & & & & & 121 & $\delta_{\mathrm{NCO}}$ & & & & & & \\
\hline 35 & $\nu_{\mathrm{OC}}$ (peptide) & & $1666 \mathrm{vw}$ & 1688 & 1693 & 1693 & 1692 & 122 & $\delta_{\mathrm{NCO}}$ & $532 \mathrm{vw}$ & $531 \mathrm{vw}$ & 566 & 584 & 566 & 564 \\
\hline 36 & $\delta_{\mathrm{NH} 2 \text { (scis) }}$ & & & 1682 & 1692 & 1671 & 1663 & 123 & $\Gamma_{\mathrm{CCNH}}+\Gamma_{\mathrm{HOCC}}$ & & & 551 & 575 & 547 & 526 \\
\hline 37 & $\delta_{\mathrm{NH} 2 \text { (scis) }}$ & 1648vs & $1636 \mathrm{~m}$ & 1626 & 1623 & 1627 & 1627 & 124 & $\Gamma_{\mathrm{CCNH}}+\Gamma_{\mathrm{HOCC}}$ & & & 547 & 565 & 529 & 525 \\
\hline 38 & $\delta_{\mathrm{NH} 2 \text { (scis) }}$ & & & 1619 & 1620 & 1626 & 1627 & 125 & $\Gamma_{\mathrm{CCNH}}$ & & & 499 & 546 & 505 & 487 \\
\hline 39 & $\delta_{\mathrm{NH} 2 \text { (scis) }}$ & & & 1613 & 1609 & 1584 & 1579 & 126 & $\Gamma_{\mathrm{CCNH}}$ & $503 w$ & $495 w$ & 494 & 538 & 503 & 485 \\
\hline 40 & $\delta_{\mathrm{NH} 2(\mathrm{scis})}$ & $1605 \mathrm{~m}$ & $1600 w$ & 1611 & 1609 & 1576 & 1578 & 127 & $\Gamma_{\mathrm{CCNH}}$ & & & 492 & 504 & 468 & 473 \\
\hline 41 & $\delta_{\mathrm{CNH}(\text { peptide) }}$ & $1527 \mathrm{~s}$ & $1525 \mathrm{~m}$ & 1511 & 1543 & 1507 & 1498 & 128 & $t_{(\mathrm{NH} 2)}+\Gamma_{\mathrm{CCNH}}$ & & & 475 & 503 & 463 & 468 \\
\hline 42 & $\delta_{\mathrm{CNH}(\text { peptide })}$ & & & 1510 & 1541 & 1493 & 1496 & 129 & $\delta_{\mathrm{NCC}}$ & $453 w$ & $452 \mathrm{w}$ & 464 & 470 & 449 & 459 \\
\hline 43 & $\delta_{\mathrm{CH} 3}$ & & & 1464 & 1461 & 1464 & 1473 & 130 & $\Gamma_{\mathrm{CCNH}}$ & & & 451 & 455 & 442 & 438 \\
\hline 44 & $\delta_{\mathrm{CH} 3}$ & & & 1463 & 1461 & 1462 & 1463 & 131 & $\delta_{\mathrm{NCC}}$ & & & 442 & 443 & 423 & 409 \\
\hline 45 & $\delta_{\mathrm{CH} 2 \text { (scis) }}$ & $1456 w$ & $1448 v s$ & 1463 & 1451 & 1460 & 1462 & 132 & $\delta_{\mathrm{CCO}}+t_{(\mathrm{NH} 2)}$ & $422 \mathrm{vw}$ & & 427 & 428 & 420 & 409 \\
\hline 46 & $\delta_{\mathrm{CH} 2 \text { (scis) }}$ & & & 1463 & 1451 & 1454 & 1459 & 133 & $\delta_{\mathrm{NCC}}$ & $419 \mathrm{vw}$ & & 422 & 422 & 407 & 393 \\
\hline 47 & $\delta_{\mathrm{CH} 2 \text { (scis) }}$ & & & 1455 & 1445 & 1453 & 1456 & 134 & $\delta_{\mathrm{NCC}}$ & & & 414 & 415 & 382 & 388 \\
\hline 48 & $\delta_{\mathrm{CH} 2 \text { (scis) }}$ & & & 1454 & 1445 & 1453 & 1452 & 135 & $r_{(\mathrm{NH} 2)}$ & & & 388 & 400 & 345 & 348 \\
\hline 49 & $\delta_{\mathrm{CH} 3}$ & $1416 \mathrm{vw}$ & $1414 \mathrm{~m}$ & 1451 & 1444 & 1450 & 1452 & 136 & $\Gamma_{\mathrm{CCNH}}$ & & $359 \mathrm{vw}$ & 345 & 393 & 329 & 334 \\
\hline 50 & $\delta_{\mathrm{CH} 3}$ & & & 1451 & 1444 & 1450 & 1452 & 137 & $\Gamma_{\mathrm{CCNH}}$ & & $350 w$ & 339 & 349 & 326 & 325 \\
\hline 51 & $\nu_{\mathrm{NC}}+\delta_{\mathrm{CCH}}$ & & & 1400 & 1402 & 1449 & 1452 & 138 & $r_{(\mathrm{NH} 2)}$ & & & 337 & 342 & 311 & 309 \\
\hline 52 & $\nu_{\mathrm{NC}}+\delta_{\mathrm{CCH}}$ & & & 1387 & 1402 & 1403 & 1436 & 139 & $\delta_{\mathrm{NCC}}$ & & & 312 & 314 & 299 & 287 \\
\hline 53 & $\delta_{\mathrm{CH} 3(\mathrm{umb})}+\delta_{\mathrm{CCH}}$ & $1404 w$ & & 1377 & 1384 & 1388 & 1375 & 140 & $\delta_{\mathrm{NCC}}$ & & & 304 & 312 & 287 & 286 \\
\hline 54 & $\delta_{\mathrm{CH} 3(\mathrm{umb})}+\delta_{\mathrm{CCH}}$ & & & 1377 & 1383 & 1377 & 1375 & 141 & $\delta_{\mathrm{CCC}}$ & & & 293 & 294 & 285 & 282 \\
\hline 55 & $\delta_{\mathrm{CH} 3(\mathrm{umb})}$ & $1380 \mathrm{~s}$ & $1375 w$ & 1371 & 1377 & 1370 & 1362 & 142 & $\delta_{\mathrm{CCC}}$ & & & 291 & 289 & 274 & 278 \\
\hline 56 & $\delta_{\mathrm{CH} 3(\mathrm{umb})}$ & & & 1370 & 1377 & 1363 & 1361 & 143 & $\Gamma_{\mathrm{CCNH}}$ & & & 271 & 276 & 270 & 268 \\
\hline 57 & $\delta_{\mathrm{NCH}} \delta_{\mathrm{CH} 3(\mathrm{umb})}$ & & $1360 w$ & 1355 & 1362 & 1361 & 1360 & 144 & $\Gamma_{\mathrm{CCNH}}$ & & & 270 & 272 & 254 & 252 \\
\hline 58 & $\delta_{\mathrm{NCH}}+\delta_{\mathrm{CH} 3(\mathrm{umb})}$ & & & 1353 & 1362 & 1357 & 1359 & 145 & $\Gamma_{\mathrm{CCNH}}$ & & & 248 & 246 & 242 & 250 \\
\hline 59 & $\delta_{\mathrm{CCH}}$ & $1350 \mathrm{vw}$ & & 1349 & 1348 & 1348 & 1354 & 146 & $\Gamma_{\mathrm{CCNH}}$ & & $248 \mathrm{vw}$ & 247 & 244 & 238 & 245 \\
\hline 60 & $\delta_{\mathrm{CCH}}$ & & & 1347 & 1348 & 1342 & 1351 & 147 & $\Gamma_{\mathrm{CCCH}}$ & & & 224 & 226 & 223 & 235 \\
\hline 61 & $\delta_{\mathrm{CCH}}$ & & & 1331 & 1333 & 1338 & 1339 & 148 & $\Gamma_{\mathrm{CCCH}}$ & & $229 w$ & 222 & 222 & 221 & 233 \\
\hline 62 & $\delta_{\mathrm{CCH}}$ & $1319 w$ & $1323 \mathrm{~m}$ & 1330 & 1333 & 1320 & 1338 & 149 & $\delta_{\mathrm{CCC}}$ & & & 216 & 218 & 212 & 206 \\
\hline 63 & $\delta_{\mathrm{CCH}}$ & $1300 w$ & & 1315 & 1311 & 1318 & 1325 & 150 & $\delta_{\mathrm{CCC}}$ & & & 215 & 206 & 207 & 206 \\
\hline 64 & $\delta_{\mathrm{CCH}}$ & & & 1312 & 1311 & 1312 & 1324 & 151 & $\delta_{\mathrm{CCC}}$ & & & 176 & 197 & 170 & 172 \\
\hline 65 & $\delta_{\mathrm{CCH}}$ & & & 1289 & 1285 & 1298 & 1302 & 152 & $\delta_{\mathrm{CCC}}$ & & & 172 & 188 & 163 & 163 \\
\hline 66 & $\delta_{\mathrm{CCH}}$ & $1281 \mathrm{vw}$ & $1279 \mathrm{~m}$ & 1288 & 1284 & 1288 & 1301 & 153 & $\delta_{\mathrm{CNC}}$ & & & 152 & 155 & 161 & 147 \\
\hline 67 & $\delta_{\mathrm{COH}}$ & & & 1254 & 1257 & 1273 & 1275 & 154 & $\delta_{\mathrm{CNC}}$ & & & 150 & 145 & 150 & 145 \\
\hline
\end{tabular}


Table 4

\begin{tabular}{|c|c|c|c|c|c|c|c|c|c|c|c|c|c|c|c|}
\hline \multicolumn{2}{|c|}{ Assign. Dimer I } & \multirow[t]{2}{*}{ IR } & \multirow[t]{2}{*}{ Raman } & \multirow{2}{*}{$\begin{array}{c}\mathrm{I} \\
1254\end{array}$} & \multirow{2}{*}{\begin{tabular}{|c|} 
II \\
1257
\end{tabular}} & \multirow{2}{*}{$\begin{array}{c}\text { III } \\
1252\end{array}$} & \multirow{2}{*}{$\begin{array}{c}\text { IV } \\
1275\end{array}$} & \multicolumn{2}{|c|}{ Assign. Dimer I } & \multirow[t]{2}{*}{ IR } & \multirow[t]{2}{*}{ Raman } & \multirow{2}{*}{$\begin{array}{c}\mathrm{I} \\
135\end{array}$} & \multirow{2}{*}{$\begin{array}{c}\text { II } \\
126\end{array}$} & \multirow{2}{*}{$\begin{array}{c}\text { III } \\
128\end{array}$} & \multirow{2}{*}{$\frac{\mathrm{IV}}{137}$} \\
\hline 68 & $\delta_{\mathrm{COH}}$ & & & & & & & 155 & $\Gamma_{\mathrm{CCCN}}$ & & & & & & \\
\hline 69 & $\delta_{\mathrm{NCH}}+\delta_{\mathrm{CCH}}$ & $1232 w$ & $1238 \mathrm{~m}$ & 1234 & 1241 & 1244 & 1242 & 156 & $\Gamma_{\mathrm{NCCN}}$ & & & 116 & 123 & 120 & 128 \\
\hline 70 & $\delta_{\mathrm{NCH}}+\delta_{\mathrm{CCH}}$ & & & 1233 & 1240 & 1241 & 1241 & 157 & $\Gamma_{\mathrm{NCCN}}$ & & & 110 & 108 & 108 & 106 \\
\hline 71 & $\delta_{\mathrm{CCH}}$ & $1214 \mathrm{vw}$ & $1212 \mathrm{~m}$ & 1221 & 1235 & 1229 & 1234 & 158 & $\Gamma_{\mathrm{CCCN}}$ & & & 103 & 103 & 104 & 103 \\
\hline 72 & $\delta_{\mathrm{CCH}}$ & $1208 \mathrm{vw}$ & & 1221 & 1232 & 1220 & 1233 & 159 & $\Gamma_{\mathrm{CCNH}}$ & & & 97 & 96 & 98 & 96 \\
\hline 73 & $\nu_{\mathrm{NC}(\text { peptide })}$ & & & 1195 & 1214 & 1209 & 1220 & 160 & $\Gamma_{\mathrm{OCCN}}$ & & & 88 & 88 & 80 & 84 \\
\hline 74 & $\nu_{\mathrm{NC}(\text { peptide })}$ & & & 1194 & 1213 & 1190 & 1219 & 161 & $\Gamma_{\mathrm{OCCN}}$ & & & 87 & 77 & 73 & 82 \\
\hline 75 & $\delta_{\mathrm{CCH}}$ & & & 1170 & 1175 & 1177 & 1182 & 162 & $\Gamma_{\mathrm{CCCN}}$ & & & 83 & 71 & 59 & 66 \\
\hline 76 & $\delta_{\mathrm{CCH}}$ & $1165 \mathrm{vw}$ & $1160 v w$ & 1168 & 1174 & 1160 & 1182 & 163 & $\Gamma_{\mathrm{CCNH}}$ & & & 71 & 63 & 58 & 61 \\
\hline 77 & $\nu_{\mathrm{OC}}+\delta_{\mathrm{COH}}$ & & & 1140 & 1140 & 1153 & 1153 & 164 & $\Gamma_{\mathrm{CCNH}}$ & & & 65 & 58 & 55 & 61 \\
\hline 78 & $\nu_{\mathrm{OC}}+\delta_{\mathrm{COH}}$ & & & 1139 & 1140 & 1144 & 1153 & 165 & $\Gamma_{\mathrm{CCCH}}$ & & & 55 & 49 & 48 & 47 \\
\hline 79 & $\nu_{\mathrm{NC}}$ & & & 1114 & 1111 & 1122 & 1118 & 166 & $\Gamma_{\text {OCCC }}$ & & & 47 & 45 & 45 & 45 \\
\hline 80 & $\nu_{\mathrm{NC}}$ & $1110 \mathrm{~m}$ & & 1110 & 1110 & 1113 & 1118 & 167 & $\Gamma_{\text {OCCC }}$ & & & 46 & 41 & 40 & 40 \\
\hline 81 & $r_{\mathrm{NH} 2}$ & & & 1107 & 1104 & 1103 & 1107 & 168 & $\Gamma_{\text {OCCC }}$ & & & 45 & 35 & 39 & 36 \\
\hline 82 & $r_{\mathrm{NH} 2}$ & & & 1101 & 1101 & 1100 & 1107 & 169 & $\Gamma_{\mathrm{CCNH}}$ & & & 40 & 35 & 33 & 30 \\
\hline 83 & $\nu_{\mathrm{NC}}$ & $1089 w$ & $1105 \mathrm{~s}$ & 1097 & 1096 & 1095 & 1067 & 170 & $\Gamma_{\mathrm{CCCH}}$ & & & 33 & 26 & 29 & 26 \\
\hline 84 & $\nu_{\mathrm{NC}}$ & & & 1096 & 1092 & 1066 & 1067 & 171 & $\Gamma_{\mathrm{OCCN}}$ & & & 29 & 21 & 21 & 25 \\
\hline 85 & $w_{(\mathrm{CH} 3)}$ & $1068 w$ & $1067 \mathrm{w}$ & 1056 & 1068 & 1063 & 1058 & 172 & $\Gamma_{\mathrm{OCCN}}$ & & & 29 & 18 & 18 & 21 \\
\hline 86 & $w_{(\mathrm{CH} 3)}$ & & & 1053 & 1068 & 1062 & 1058 & 173 & $\Gamma_{\mathrm{OCCN}}$ & & & 22 & 14 & 13 & 16 \\
\hline 87 & $\nu_{\mathrm{CC}}$ & $1044 w$ & $1043 w$ & 1021 & 1030 & 1019 & 1020 & 174 & $\Gamma_{\mathrm{OCCN}}$ & & & 20 & 8 & 8 & 12 \\
\hline
\end{tabular}

Notes: * The wavenumbers under $1800 \mathrm{~cm}^{-1}$, were scaled either with 0.967 (for B3LYP/6-31G(d,p)) or 0.977 (for B3LYP/6-31++G(d,p)), and for over $1800 \mathrm{~cm}^{-1}$ the scale factor 0.955 were used for both B3LYP/6-31G(d,p), B3LYP/6-31++G(d,p) levels of theory. 
0.955. The scaled frequencies of Ala-Gln are well consistent with the experimental data. The stretching vibrations of methylene and methine group of Ala-Gln are assigned between 3000 and $2874 \mathrm{~cm}^{-1}$ as sharp bands (see Table 3). The symmetrical $\mathrm{CH}_{3}$ stretching vibration is observed in IR as a weak intense band at $2877 \mathrm{~cm}^{-1}$ and in Raman spectrum as a medium band at $2874 \mathrm{~cm}^{-1}$. The asymmetrical $\mathrm{CH}_{3}$ stretching is assigned as a strong band at $3225 \mathrm{~cm}^{-1}$ in the IR spectrum and a weak band at $3224 \mathrm{~cm}^{-1}$ in the Raman spectrum.

The inter and intra hydrogen bonding differences between mono Ala-Gln and Dimer I are found to reflect in the calculated wavenumbers of mono and Dimer I structures (Table 3).

\section{Conclusion}

The monomer and dimer structures of Ala-Gln were studied by using the DFT/B3LYP method with $6-31++\mathrm{G}(\mathrm{d}, \mathrm{p})$ basis set. The structural characteristics of these molecules were revealed. Purpose of this work is to investigate vibrational modes of the energetically most stable conformer of mono- and dimer Ala-Gln molecules. We calculated 810 conformers for mono-Ala-Gln dipeptide and four dimer conformers (I-IV) for dimmer-Ala-Gln molecule. The mono-Ala-Gln dipeptide are characterized by the extended backbone shape in the LB conformational range [11,12] with the energy $-0.18 \mathrm{kcal} / \mathrm{mol}$. The most stable conformers of dimer-Ala-Gln molecule (Dimer I) has $E=-1558.25542661$ a.u. This investigation provides a rather satisfactory theoretical description of the monomer and dimer structures of Ala-Gln molecules. The determination of conformational details of biological macromolecules and conformational possibilities of Ala-Gln dipeptide is very important to understand their functions of a drug and may be useful as a base for synthesis of their more effective structural analogs.

\section{Acknowledgement}

This study was supported by the Research fund of Istanbul University (project Nos are T/3171 and ONAP-2423).

\section{References}

[1] L.C.A. Cerchietti, A.H. Navigante, M.A. Lutteral, M.A. Castro, R. Kirchuk, M. Bonomi, M.E. Cabalar, B. Roth, G. Negretti, B. Sheinker and P. Uchima, Int. J. Radiat. Oncol. Biol. Phys. 65(5) (2006), 1330.

[2] C. Decker-Baumann, K. Buhl, S. Frohmüller, A.V. Herbay, M. Dueck and P.M. Shlag, Eur. J. Cancer 35 (1999), 202.

[3] H. Eagle, V.I. Oyama, M. Levy, C.L. Horton and R. Fleischman, J. Biol. Chem. 218 (1956), 607.

[4] M.J. Frisch, G.W. Trucks, H.B. Schlegel, G.E. Scuseria, M.A. Robb, J.R. Cheeseman, J.A. Montgomery Jr., T. Vreven, K.N. Kudin, J.C. Burant, J.M. Millam, S.S. Iyengar, J. Tomasi, V. Barone, B. Mennucci, M. Cossi, G. Scalmani, N. Rega, G.A. Petersson, H. Nakatsuji, M. Hada, M. Ehara, K. Toyota, R. Fukuda, J. Hasegawa, M. Ishida, T. Nakajima, Y. Honda, O. Kitao, H. Nakai, M. Klene, X. Li, J.E. Knox, H.P. Hratchian, J.B. Cross, V. Bakken, C. Adamo, J. Jaramillo, R. Gomperts, R.E. Stratmann, O. Yazyev, A.J. Austin, R. Cammi, C. Pomelli, J.W. Ochterski, P.Y. Ayala, K. Morokuma, G.A. Voth, P. Salvador, J.J. Dannenberg, V.G. Zakrzewski, S. Dapprich, A.D. Daniels, M.C. Strain, O. Farkas, D.K. Malick, A.D. Rabuck, K. Raghavachari, J.B. Foresman, J.V. Ortiz, Q. Cui, A.G. Baboul, S. Clifford, J. Cioslowski, B.B. Stefanov, G. Liu, A. Liashenko, P. Piskorz, I. Komaromi, R.L. Martin, D.J. Fox, T. Keith, M.A. AlLaham, C.Y. Peng, A. Nanayakkara, M. Challacombe, P.M.W. Gill, B. Johnson, W. Chen, M.W. Wong, C. Gonzalez and J.A. Pople, Gaussian03, Revision C.02, Gaussian, Inc., Wallingford, CT, 2004.

[5] S. Ghosh, S. Mondal, A. Misra and S. Dalai, J. Mol. Struct. 805 (2007), 133.

[6] N.M. Godjaev, I.S. Maksumov and I. Ismailoval, J. Chem. Struct. 24 (1983), 147.

[7] S. Mondal, D.S. Chowdhuri, S. Ghosh, A. Misra and S. Dalai, J. Mol. Struct. 810 (2007), 81. 
[8] K. Nakamoto, Infrared Spectra of Inorganic and Coordination Compounds, Wiley, London, 1970.

[9] J. Ockenga, K. Borchert, K. Rifai, M. Petermanns and S. Bischoff, Efect of glutamine-enriched total parenteral nutrition in patients with acute pancreatitis, Clin. Nutr. 21(5) (2002), 409.

[10] PQS Version 3.1, Parallel Quantum Solutions, 2013 Green Acres Road, Fayetteville, AR.

[11] G.N. Ramachandran, Biopolymers 6 (1963), 1494.

[12] G.N. Ramachandran, C. Ramakrishnan and V. Sasisekharan, J. Mol. Biol. 7 (1963), 95.

[13] D.M.F. Savarese, G. Savy, L. Vahdat, P.E. Wischmeyer and B. Corey, Prevention of chemotherapy and radiation toxicity with glutamine, Cancer Treat. Rev. 29 (2003), 501.

[14] K.M. Skubitz and P.M. Anderson, Oral glutamine to prevent chemotherapy induced stomatitis: A pilot study, J. Lab. Clin. Med. 127(2) (1996), 223.

[15] H.C.T. van Zaanen, H. van der Lelie, J.G. Timmer, P. Fürst and H.P. Sauerwein, Cancer 74(10) (1994), 2879. 


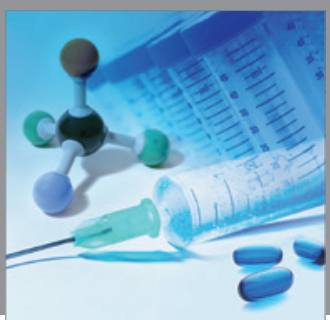

International Journal of

Medicinal Chemistry

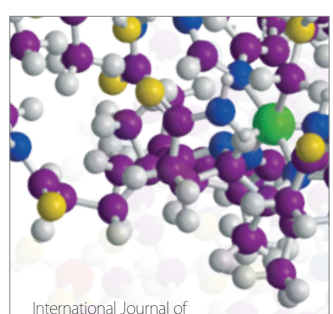

Carbohydrate Chemistry

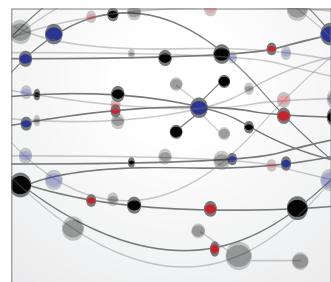

The Scientific World Journal
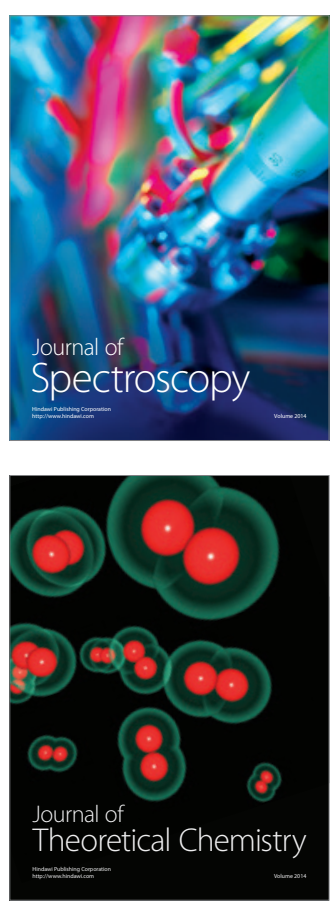
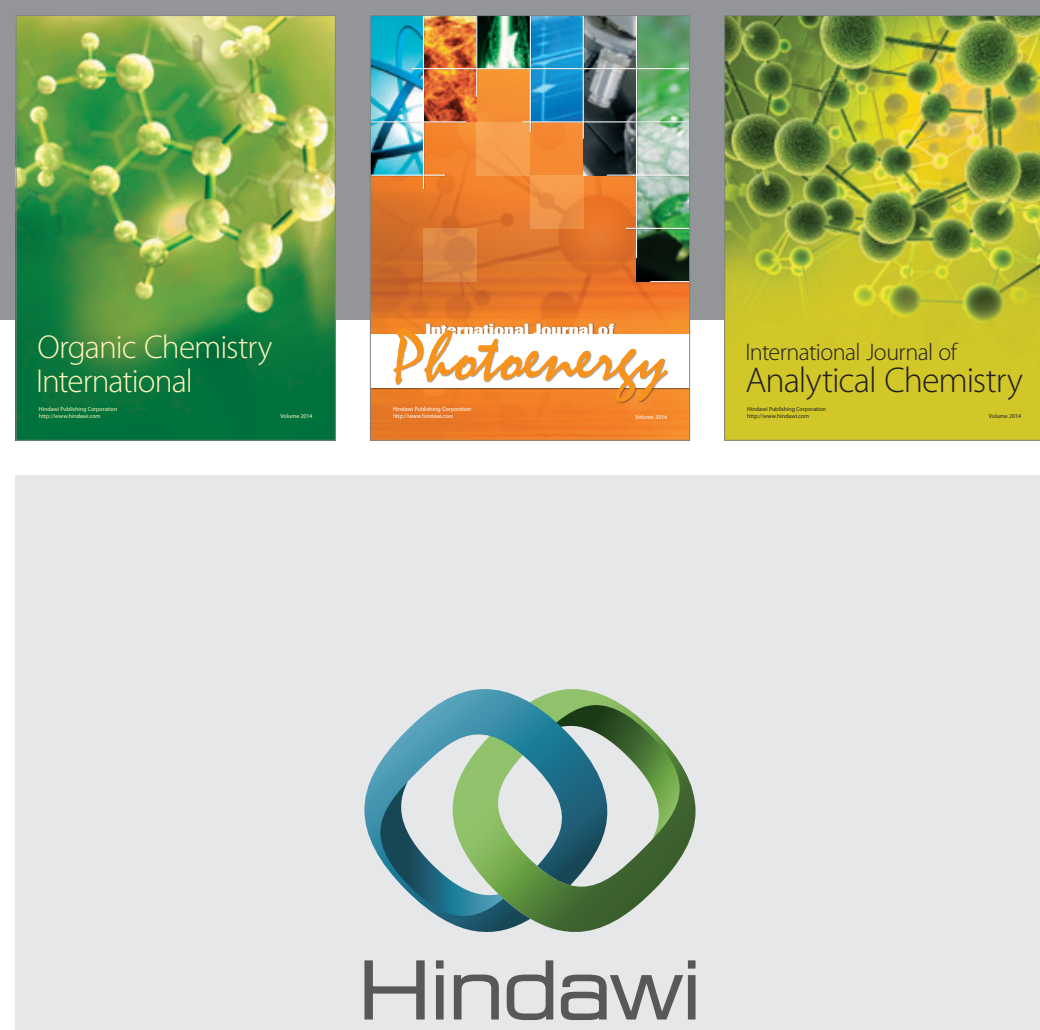

Submit your manuscripts at

http://www.hindawi.com
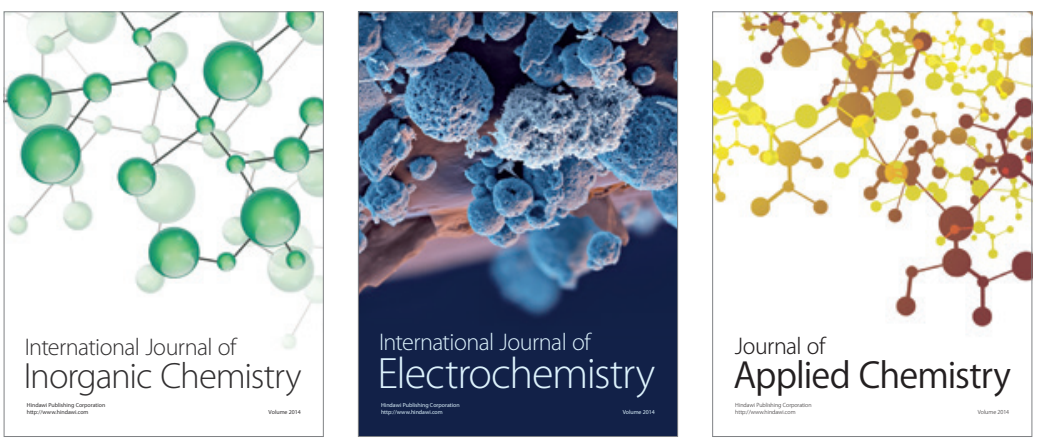

Journal of

Applied Chemistry
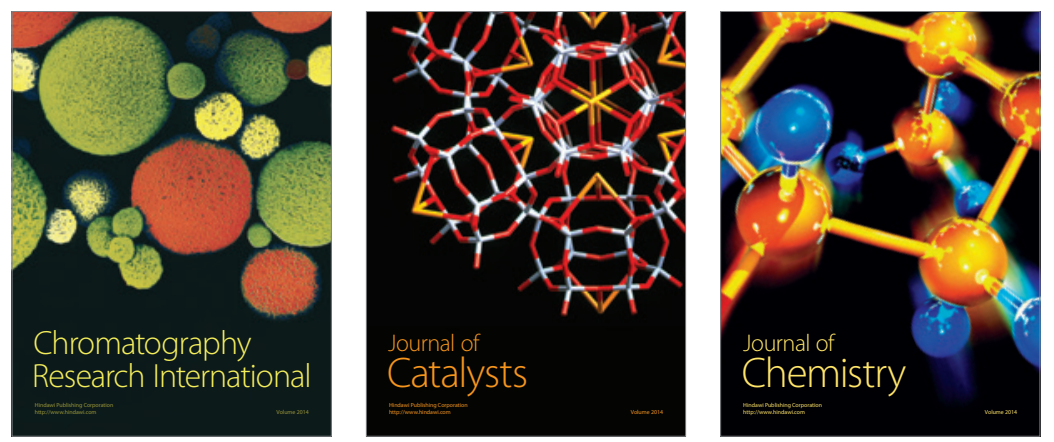
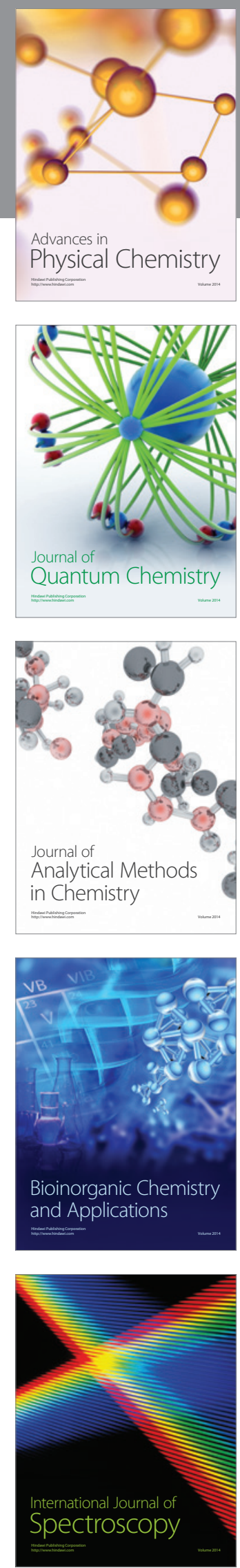